\title{
Wellens' Syndrome: An Atypical Presentation of an Already Silent Killer
}

\author{
Muhammad Mufti $^{\mathrm{a}, \mathrm{c}}$, Rajveer Joea ${ }^{\mathrm{a}}$, Serap Sobnosky ${ }^{\mathrm{b}}$, Jana Longtine ${ }^{\mathrm{a}}$
}

\begin{abstract}
Wellens' syndrome is a phenomenon of T-wave inversions seen in the precordial leads of the electrocardiogram in the patients with unstable angina. This finding is seen in the pain-free state. Two different patterns have been described. Type A presents with biphasic $\mathrm{T}$ waves in V2-V3 and type B presents with symmetrical deep T-wave inversions in V2-V3. This etiology signifies critical stenosis in proximal left anterior descending artery (LAD). We describe a patient who was admitted for an elective surgery without a history of chest pain noted to have Wellens pattern T-wave inversions on telemetry and found to have $60-70 \%$ stenosis in mid LAD.
\end{abstract}

Keywords: Wellens' syndrome; Left anterior descending artery; Stenosis; Chest pain; Unstable angina

\section{Introduction}

Wellens' syndrome is a phenomenon of T-wave inversions noted in the precordial leads of patients with unstable angina. These patients were originally described to have critical stenosis of the proximal left anterior descending artery (LAD). If untreated, it progresses to anterior myocardial infarction in the majority of the cases. We will present a variant case of Wellens' syndrome.

\section{Case Report}

An 87-year-old female was admitted to the hospital for an elective skin grafting procedure by plastic surgery. She had recently fallen and subsequently developed a hematoma in the right lower extremity. Her medical history was significant for hypertension, hyperlipidemia, paroxysmal atrial fibrillation

Manuscript submitted March 17, 2018, accepted April 30, 2018

aDepartment of Medicine, St. Mary Medical Center, Long Beach, CA, USA bDepartment of Cardiology, St. Mary Medical Center, Long Beach, CA, USA cCorresponding Author: Muhammad Mufti, Department of Medicine, St. Mary Medical Center, GME, 1050 Linden Ave., Long Beach, CA 90813, USA. Email: muneebmufti71@gmail.com

doi: https://doi.org/10.14740/jmc3058e (on rivaroxaban), ischemic stroke without any residual deficits and malignant melanoma status post resection and axillary lymph node dissection. The patient had a remote smoking history. The patient denied any history of chest pain. Her admission blood pressure was 192/110 mm Hg. Examination on admission revealed normal S1 and S2 heart sound without any murmurs. The patient had a scar of a sternotomy, but she could not recall what type of surgery she had in the past. Hospital records did not have any information either. Admission electrocardiogram (EKG) showed atrial fibrillation with left axis deviation at heart rate of 84 beats per minute (Fig. 1). The patient was admitted to telemetry floor, and her blood pressure was controlled with medications.

Overnight dynamic changes in the telemetry were noted. Repeat EKG was performed (Fig. 2) which showed patient in sinus rhythm with a rate of 75 beats per minute with left axis deviation and T-wave inversions in lead V2 to V6, I and aVL. Less than 1-mm ST-segment depression was seen in V6 and lead I. Troponins were drawn and were negative two times. The patients' EKG was concerning for Wellens' syndrome, but the patient did not have a history of chest pain. Due to her risk factors and recent dynamic changes, it was decided that cardiac catheterization should be offered.

The patient consented to the procedure, and catheterization revealed that she had no significant stenosis in the left main coronary artery, left circumflex artery, and right coronary artery. The patient had $60-70 \%$ stenosis in mid LAD (Fig. 3). Fractional flow reserve (FFR) without adenosine was calculated in the lesion, which was 0.83 , and the cutoff used at our institution is 0.9 . Hence the decision was made to stent the lesion. Two drug-eluting stents (DESs) were deployed in the lesions (Fig. 4 ), and the patient was started on aspirin and ticagrelor. The patient was given an outpatient follow-up with a cardiologist.

\section{Discussion}

Wellens' syndrome was first described by De Zwann et al in a study group of 145 patients with unstable angina, and 26 patients had the EKG pattern of Wellens' syndrome [1]. Twelve patients out of this group did not undergo coronary revascularization and subsequently developed anterior myocardial infarction within a few weeks' time. Later on, a larger sample was used (1,260 patients, 180 patients with Wellens EKG findings) and all of the patients who showed these EKG changes were found to have stenosis in the proximal LAD ranging from $50 \%$ to $100 \%$ [2]. The causes of T-wave inversion can be ex- 


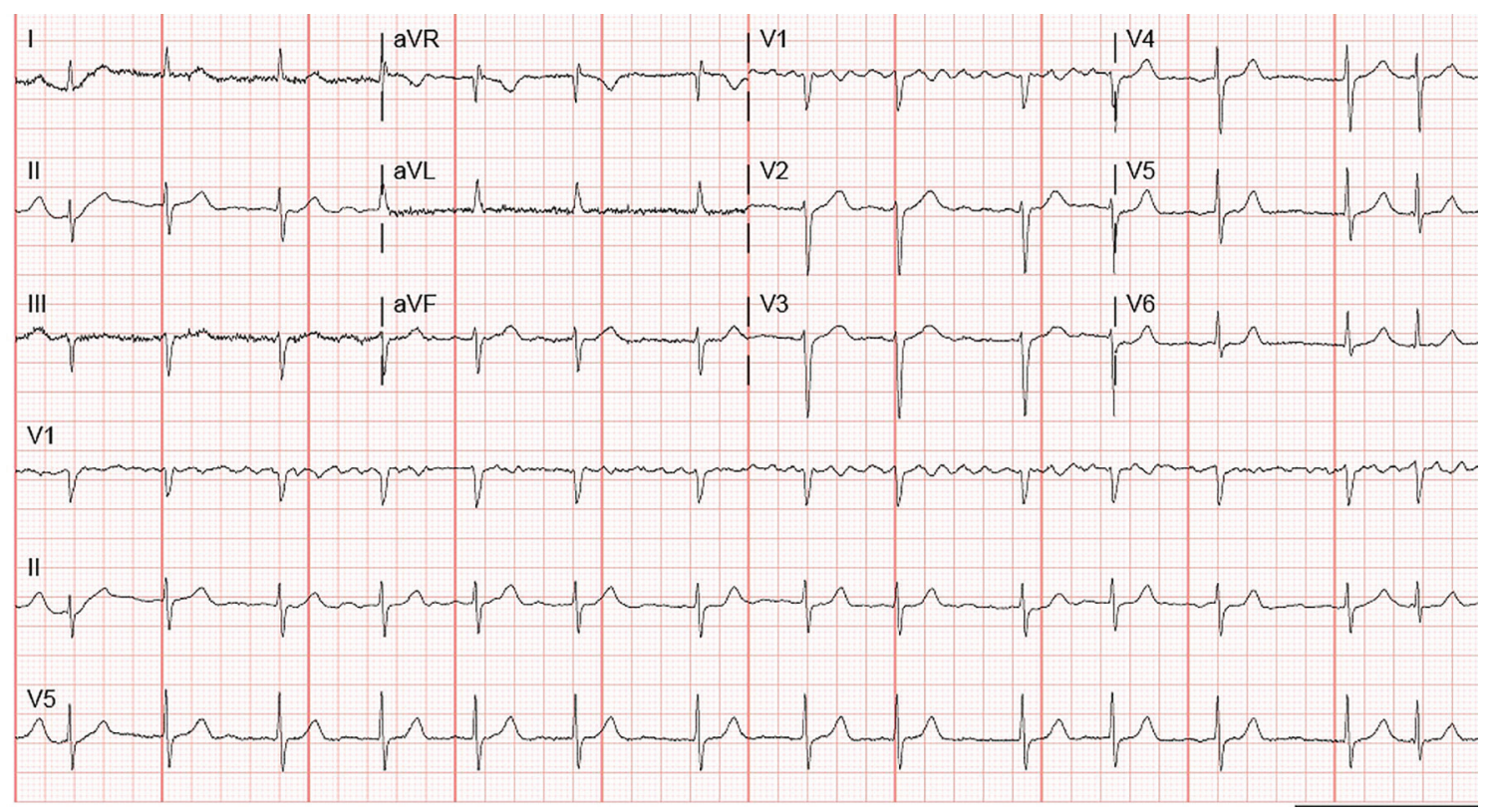

Figure 1. Admission EKG showing atrial fibrillation.

tensive and include coronary ischemia and pulmonary embolism, myocarditis, later stage of pericarditis, myocardial straining following ventricular hypertrophy, bundle branch block, pre-excitation syndrome, and digitalis effect [3]. Rhinehardt et al described the following diagnostic criteria for Wellens' syndrome [4]: 1) deeply inverted or biphasic $\mathrm{T}$ waves in V2-3 (may extend to V1-6); 2) isoelectric or minimally elevated STsegment ( $<1 \mathrm{~mm})$; 3) no precordial Q waves; 4) preserved precordial $\mathrm{R}$ wave progression; 5) a recent history of angina; 6) ECG pattern present in the pain-free state; 7) normal or slightly elevated serum cardiac markers.

Usually, two different patterns of $\mathrm{T}$ wave abnormality are seen in Wellen's syndrome type A and type B. Type A has biphasic $\mathrm{T}$ waves with initial positivity and terminal negativity (it was seen in about $25 \%$ of the cases) [1]. Type B has T waves which are deep and symmetrically inverted (it was seen in about $75 \%$ of the cases) [1]. This syndrome was described to represent critical stenosis of the proximal LAD.

Throughout the years, many cases have been reported with typical and atypical features of Wellens' syndrome. Kardesog-

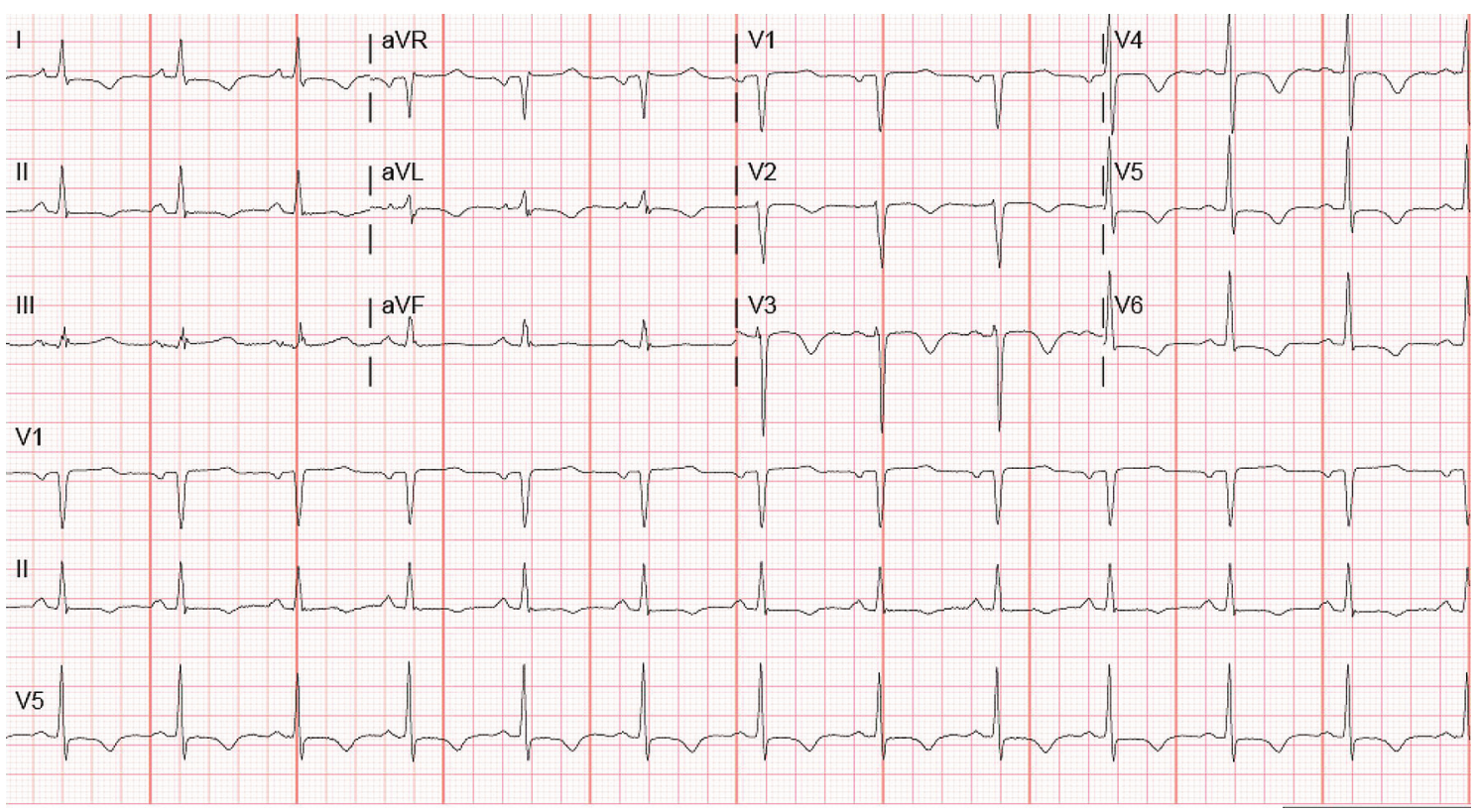

Figure 2. EKG performed after dynamic changes noted on telemetry showing sinus rhythm with rate of 75 beats per minute with left axis deviation, T-wave inversions in lead V2 to V6, I and aVL and less than 1-mm ST-segment depression in V6 and lead I. 


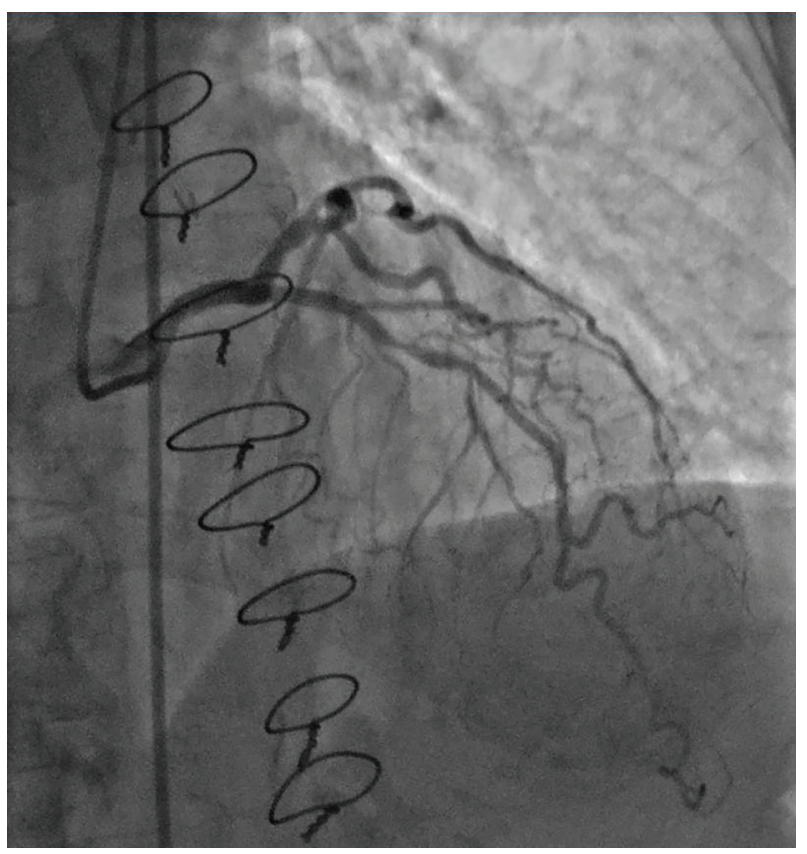

Figure 3. Angiogram showing mid LAD lesion with $60-70 \%$ stenosis.

lu et al reported a case where the patient had T-wave inversions in the lead II, III and aVF and the EKG changed from type B to type A pattern on serial EKGs. This patient also had the unusual finding that the critical stenosis was in mid LAD [5]. Taylor et al reported a case of 65-year-old female with a history of seizure disorder presenting with EKG of type A Wellens but cardiac catheterization showing Takotsubo cardiomyopathy [6]. Others have tried to diagnose Wellens in the presence of left bundle branch block (LBBB). Grautoff presented a case of LBBB and chest pain. Sgarbossa's criteria were not met, and the patient was admitted for monitoring. Serial EKG showed type A Wellens. The patient was taken to cardiac catheterization showing mid LAD stenosis [7]. Kyaw et al reported an elderly female with throat pain as the presentation of an acute coronary syndrome and EKG findings of Wellens and revealing proximal LAD lesion on catheterization [8]. Some cases of pseudo-Wellens have been described with the use of cocaine and marijuana $[9,10]$. Another case study described a case of Wellens with T-wave changes during the pain episode as well as the pain-free periods [11].

The case we presented above highlights that there can be variants in Wellens' syndrome. The patients' presentation was atypical, and the lesion was in mid LAD instead of the classical proximal LAD lesion. She was admitted to the hospital for an elective procedure of skin grafting by plastic surgery. She did not have any chest pain or angina equivalent. Troponins drawn before catheterization were normal twice. EKG done on admission showed atrial fibrillation with left axis deviation at heart rate of 84 beats per minute. Second EKG done about 14 $\mathrm{h}$ later showed patient in sinus rhythm with rate of 75 beats per minute with left axis deviation, T-wave inversions in lead V2 to V6, I and aVL and less than 1-mm ST-segment depression in V6 and lead I. Third EKG was performed about $5 \mathrm{~h}$ later which did not show any T-wave inversions. Due to the concern for

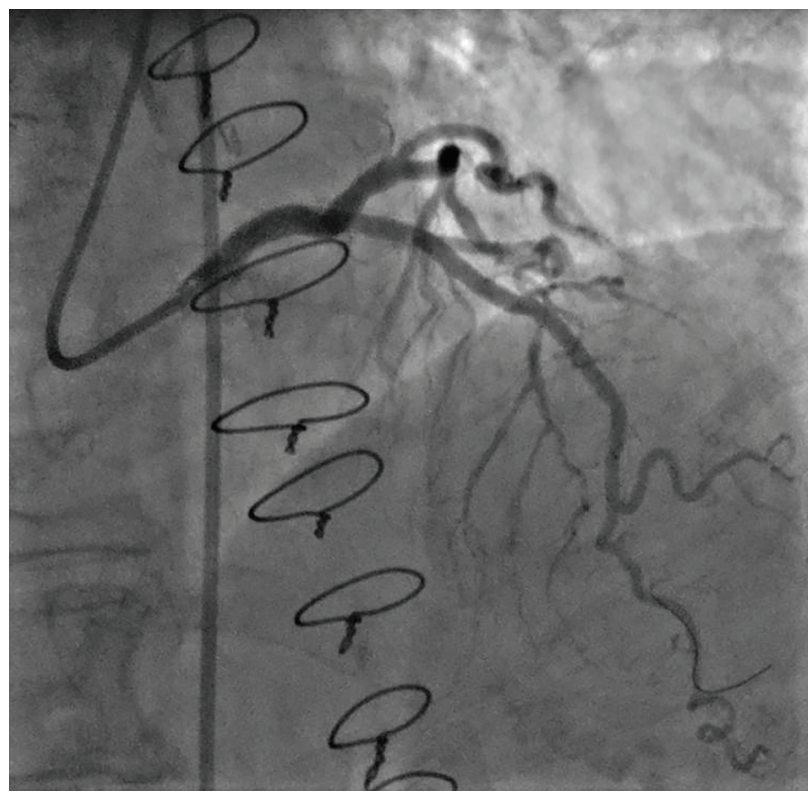

Figure 4. Angiogram after placement of drug-eluting stents showing no residual stenosis.

type B Wellens' syndrome, the patient was taken for cardiac catheterization which showed two areas of significant stenosis around $60-70 \%$ in mid portion of the LAD. Left main coronary artery, left circumflex artery, and right coronary artery had no significant stenosis. FFR calculation being abnormal was a factor in the decision of stenting the lesion.

\section{Conclusion}

Wellens' syndrome is a pattern of T-wave changes in the precordial leads that occurs in a subset of patients with unstable angina signifying stenosis of proximal LAD. It can have an atypical presentation, and our case highlights a patient with EKG characteristics of Wellens' but stenosis of mid LAD instead of proximal. More studies need to be done to fully understand the different locations of lesions and their associated EKG findings. Early treatment of Wellens' syndrome prevents the patient from developing myocardial infarction.

\section{References}

1. de Zwaan C, Bar FW, Wellens HJ. Characteristic electrocardiographic pattern indicating a critical stenosis high in left anterior descending coronary artery in patients admitted because of impending myocardial infarction. Am Heart J. 1982;103(4 Pt 2):730-736.

2. de Zwaan C, Bar FW, Janssen JH, Cheriex EC, Dassen WR, Brugada P, Penn OC, et al. Angiographic and clinical characteristics of patients with unstable angina showing an ECG pattern indicating critical narrowing of the proximal LAD coronary artery. Am Heart J. 1989;117(3):657665. 
3. Yub Raj Sedhai, Aditya Singh Pawar, Priyandka T. Bhattacharya, et al. Wellens' sydnrome, a case report of an electrographic warning sign. Journal of Advances in Internal Medicine. 2016;05(01):19-23.

4. Rhinehardt J, Brady WJ, Perron AD, Mattu A. Electrocardiographic manifestations of Wellens' syndrome. Am J Emerg Med. 2002;20(7):638-643.

5. Kardesoglu E, Celik T, Cebeci BS, Cingozbay BY, Dincturk M, Demiralp E. Wellens' syndrome: a case report. J Int Med Res. 2003;31(6):585-590.

6. Taylor RS, Skjerli L, Ashurst J. Takotsubo Cardiomyopathy Presenting as Wellens' Syndrome. CPC-EM: volume 1 issue 3.

7. Grautoff S. Wellens' syndrome can indicate high-grade
LAD stenosis in case of left bundle branch block. Herzschrittmacherther Elektrophysiol. 2017;28(1):57-59.

8. Kyaw K, Latt H, Aung SSM, Tun NM, Phoo WY, Yin HH. Atypical presentation of acute coronary syndrome and importance of Wellens' syndrome. Am J Case Rep. 2018;19:199-202.

9. Lin AN, Lin S, Gokhroo R, Misra D. Cocaine-induced pseudo-Wellens' syndrome: a Wellens' phenocopy. BMJ Case Rep. 2017;2017(

10. Co MLF, Das A, Okwuosa T. Pseudo-Wellens syndrome after heavy marijuana use. Cleve Clin J Med. 2017;84(8):590-591.

11. Sobnosky S, Kohli R, Bleibel S. Wellens' Syndrome. The Internet Journal of Cardiology. 2005;3(1). 